\title{
P64 Carotid Artery Tracking with Automated Wall Position Resets Yields Robust Distension Waveforms in Long-term Ultrasonic Recordings
}

\author{
Fabian Beutel ${ }^{1,2,{ }^{*}}$, Laura Mansilla Valle ${ }^{1,3}$, Chris Van Hoof ${ }^{2,4}$, Evelien Hermeling ${ }^{1}$ \\ ${ }^{1}$ imec, Eindhoven, The Netherlands \\ ${ }^{2}$ KU Leuven, Leuven, Belgium \\ ${ }^{3}$ University of Vic, Vic, Catalonia \\ ${ }^{4}$ imec, Leuven, Belgium
}

\begin{abstract}
Background: Carotid artery tracking has high clinical relevance for the investigation of arterial stiffness indicators like Pulse Wave Velocity (PWV). However, current tracking systems are unreliable and/or not fully automated [1,2]. In this work we propose a novel wall tracking algorithm for long-term ultrasonic recordings, featuring automated beat-to-beat end-diastolic wall position resets.

Methods: Carotid artery ultrasound (Vantage64, Verasonics, USA) and simultaneous ECG (ECG100C, BIOPAC, USA) were acquired from 10 subjects ( $38 \pm 10$ years) in 6 repeated measurements, each involving a resting, breathing and handgrip intervention. The ECG triggers an automated algorithm, whose heuristics utilize the hypoechogenic lumen to detect the enddiastolic wall positions in the ultrasound data. Subsequently, wall motion is tracked throughout the cardiac cycle by complex cross-correlation [3]. Further processing yields carotid distension waveforms and local PWV via spatiotemporal fitting of waveform fiducials. The novel per-beat algorithm was benchmarked against a manually initialized per-intervention algorithm, while ground truth wall positions were manually annotated. Performance was assessed for temporal efficiency, spatial accuracy and feature consistency.
\end{abstract}

Results: Average results show a $~ 4000 \%$ higher temporal efficiency, $20 \%$ increased spatial accuracy ( $\mu$ error: 0.66 to 0.53 [mm]) and $14 \%$ improved feature consistency (oPWV: 2.2 to $1.9[\mathrm{~m} / \mathrm{s}]$ ) for the per-beat algorithm. Results of exceptional cases reveal even more significant performance, e.g. $60 \%$ increased spatial accuracy ( $\mu$ error: 1.57 to 0.64 [mm]) for gradual drift and $58 \%$ improved feature consistency (oPWV: 1.9 to $0.8[\mathrm{~m} / \mathrm{s}]$ ) for instant vessel loss (see Figure 1).

Conclusion: The proposed algorithm demonstrates significant temporal efficiency, spatial accuracy and feature consistency, particularly during perturbations. Such robustness is essential for long-term monitoring, making the algorithm a powerful tool in ambulatory vascular research.

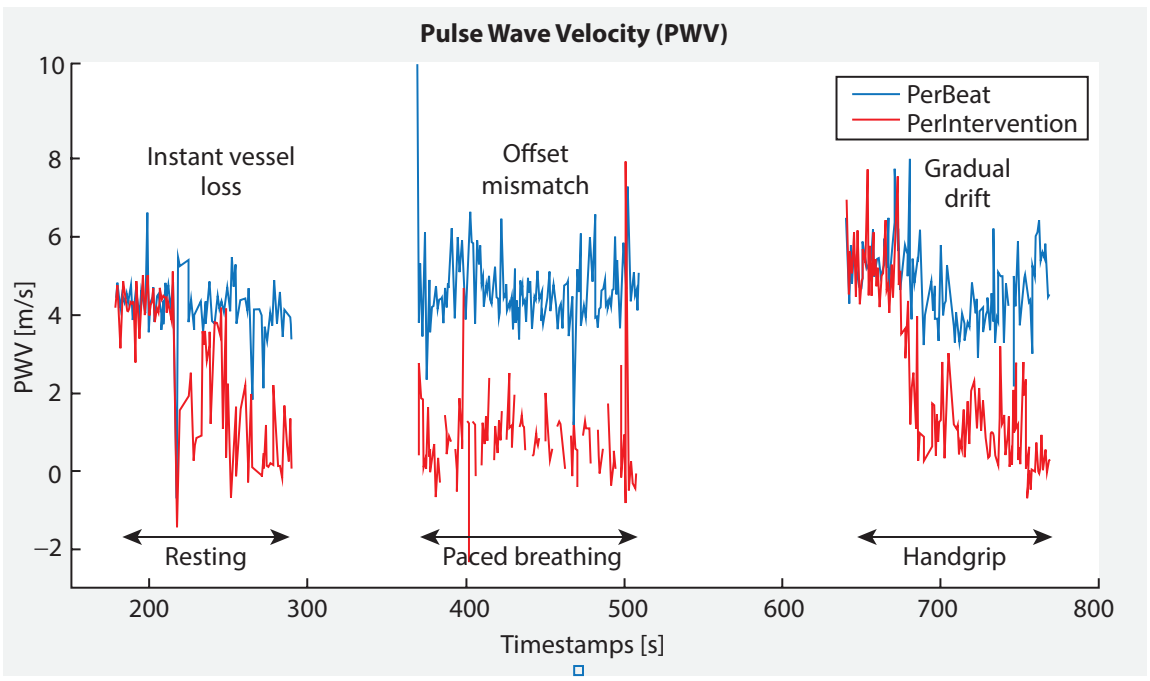

Figure 1 


\section{REFERENCES}

[1] Gao Z, Li Y, Sun Y, Yang J, Xiong H, Zhang H, et al. Motion tracking of the carotid artery wall from ultrasound image sequences: a nonlinear state-space approach. IEEE Trans Med Imaging 2018;37:273-83.

[2] Persson M, Ryden Ahlgren A, Eriksson A, Jansson T, Persson HW, Lindstrom K. Non-invasive measurement of arterial longitudinal movement. 2002 IEEE Ultrasonics Symposium, 2002. Proceedings, Munich, Germany: IEEE; 2002, pp. 1783-6.

[3] Brands PJ, Hoeks APG, Ledoux LAF, Reneman RS. A radio frequency domain complex cross-correlation model to estimate blood flow velocity and tissue motion by means of ultrasound. Ultrasound Med Biol 1997;23:911-20.

(c) 2019 Association for Research into Arterial Structure and Physiology. Publishing services by Atlantis Press International B.V. This is an open access article distributed under the CC BY-NC 4.0 license (http://creativecommons.org/licenses/by-nc/4.0/). 